\title{
Conditioning intensity and antilymphocyte globulin: towards personalized transplant strategies?
}

\section{Martin Bornhäuser}

Department of Internal Medicine I, University Hospital Carl Gustav Carus, TU Dresden and National Center for Tumor Disease (NCT), Germany

E-mail: MARTINBORNHÄUSER - martin.bornhaeuser@uniklinikum-dresden.de

doi:10.3324/haematol.2019.216952

$\mathrm{I}^{\mathrm{n}}$ the last two decades allogeneic hematopoietic cell transplantation (HCT) has been used with increasing frequencies in hematologic malignancies with curative intent. The increased understanding of immune tolerance and allogeneic antileukemic immune reactivity has led several investigators to develop optimized conditioning protocols and new strategies to manipulate the effector cells either within the graft or in vivo.

More specifically, the development of minimal intensity or so called "nonmyeloablative" conditioning regimens paved the way towards the application of allogeneic HCT in older patients and all of those who probably would not tolerate classical intensity conditioning., ${ }^{1,2}$ The most frequently used protocol, spear-headed by investigators from the Fred Hutchinson Cancer Research Center, was based on a single dose of 200 cGy of total-body irradiation (TBI) and a few doses of fludarabine followed by pharmacological immunosuppression. ${ }^{3}$ After a wave of fascinating reports on the feasibility and efficacy of this "revolutionary" approach, some studies revealed that patients with a high risk of either disease recurrence or non-engraftment did not fare too well with this strategy. ${ }^{4}$ At the other end of the spectrum, randomized comparisons suggested that less toxic but still intensive conditioning with 800 cGy of fractionated TBI combined with fludarabine did not increase the rate of disease recurrence at the same time as significantly reducing extramedullary toxicity compared to 1200 cGy TBI with high-dose cyclophosphamide..$^{5}$ Along these lines, Monaca and co-workers, ${ }^{6}$ again from Seattle, have convincingly demonstrated in this issue of Haematologica that subtle dose increases of unfractionated TBI can significantly decrease the failure rate after allogeneic HCT. Most interestingly, applying a differential dose escalation strategy, they identified the optimal TBI dose for patients with high-risk myelodysplastic syndromes and chronic myelomonocytic leukemia (450 cGy) and patients with low-risk myelodysplastic syndromes and myeloproliferative neoplasms (300 cGy). Intermediate doses of unfractionated TBI have been successfully used by other colleagues in diseases such as chronic myeloid leukemia. ' The current optimization of the nonmyeloablative protocol developed by the investigators in Seattle clearly shows that differential doses of TBI complement the most frequently applied protocols based on alkylating agents combined with purine analogs.

As stated above, the fine tuning of the allogeneic immune response by $\mathrm{T}$-cell depletion or pharmacological means rep-

Toolbox

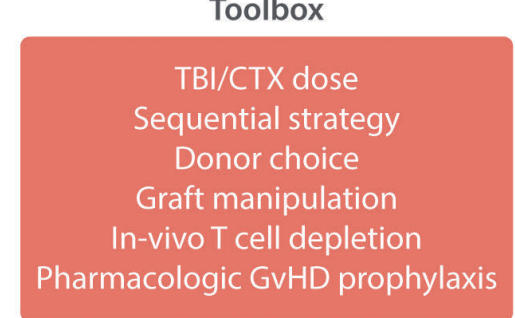

- Disease characteristics (incl. molecular profiling)

- Patient status/comorbidities (pharmacogenetics)

- Immunogenetic data (permissive mismatches etc.)

- Patient's expectations

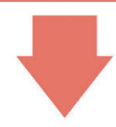

Data integration algorithm

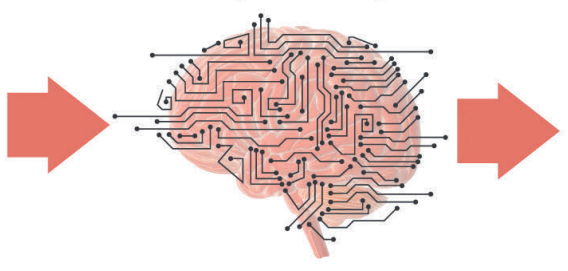

Personalized HCT approach

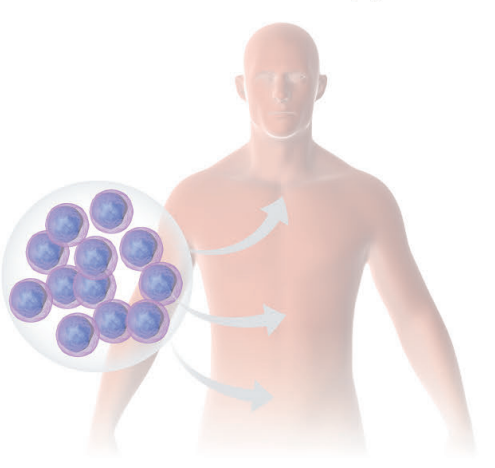

Figure 1. A personally tailored approach to hematopoietic cell transplantation. TBI; total body irradiation; CTX: chemotherapy; GvHD: graft-versus-host disease; HCT: hematopoietic cell transplantation. 
resents the second important pillar in the development of optimized transplantation protocols. Specifically, the use of antihuman T lymphocyte globulin (ATG), a polyvalent preparation generated in rabbits against Jurkat $T$ cells, was introduced several decades ago. Antithymocyte gobulin, derived from rabbits or horses against human thymocytes, has predominantly been used for the treatment of severe aplastic anemia but also within conditioning for allogeneic HCT in selected protocols, mainly for non-malignant indications. ${ }^{8}$ The pivotal trial testing ATG in the setting of unrelated donors and intensive conditioning suggested a significant reduction in the incidence of chronic graft-versus-host disease without an increase in the risk of relapse. ${ }^{9}$ While this trial tested a dose of $60 \mathrm{mg} / \mathrm{kg}$ ATG (day -3 to -1 ), a similar finding was made after allogeneic HCT from matched siblings applying $30 \mathrm{mg} / \mathrm{kg}$ again within intensive conditioning. ${ }^{10}$ Both trials included patients with acute leukemia and myelodysplastic syndromes. As a note of caution, a second controlled trial performed in the unrelated donor setting did not confirm the initial observations, suggesting a potential interaction between the choice of conditioning regimen and the chosen ATG regimen. ${ }^{11}$ So far, no randomized trial had formally tested ATG after reduced intensity conditioning in transplants for a specific indication. Robin and coworkers now present the results of a retrospective analysis performed in patients with myelofibrosis receiving reduced intensity conditioning. ${ }^{12}$ Interestingly, ATG reduced the cumulative incidence of acute graft-versus-host disease while it did not affect the rate of chronic graft-versus-host disease. Although the authors speculate that this may be due to the use of reduced intensity conditioning, there might be various other factors that could lead to differential effects in this specific group of patients, most of whom still have relatively high lymphocyte counts and organomegaly which can influence the pharmacodynamics of ATG. ${ }^{13}$

Although both trials address different aspects in the effort to optimize outcomes after allogeneic HCT, they clearly demonstrate that patient and disease characteristics but also graft source and choice of donor can significantly affect the outcome of treatment. Integrating the mentioned factors, the current toolbox offers conditioning protocols of almost all intensities including sequential regimens ${ }^{14}$ as well as various options of modulating allogeneic immune responses such as by graft manipulation or in vivo T-cell depletion. Taking advantage of all available knowledge gained by artificial intelligence and large database ${ }^{15}$ may enable design of the ideal preparative regimen and post-grafting immunosuppression for a given patient and in a specific immunogenetic setting thereby making allogeneic HCT a tailored approach comparable to that of several other current strategies in modern oncology (Figure 1).

\section{References}

1. Giralt S, Estey E, Albitar M, et al. Engraftment of allogeneic hematopoietic progenitor cells with purine analog-containing chemotherapy: harnessing graft-versus-leukemia without myeloablative therapy. Blood. 1997;89(12):4531-4536.

2. Slavin S, Nagler A, Naparstek E, et al. Nonmyeloablative stem cell transplantation and cell therapy as an alternative to conventional bone marrow transplantation with lethal cytoreduction for the treatment of malignant and nonmalignant hematologic diseases. Blood. 1998;91(3):756-763.

3. McSweeney PA, Niederwieser D, Shizuru JA, et al. Hematopoietic cell transplantation in older patients with hematologic malignancies: replacing high-dose cytotoxic therapy with graft-versus-tumor effects. Blood. 2001;97(11):3390-3400.

4. Sorror ML, Sandmaier BM, Storer BE, et al. Long-term outcomes among older patients following nonmyeloablative conditioning and allogeneic hematopoietic cell transplantation for advanced hematologic malignancies. JAMA. 2011;306(17):1874-1883.

5. Bornhauser M, Kienast J, Trenschel R, et al. Reduced-intensity conditioning versus standard conditioning before allogeneic haemopoietic cell transplantation in patients with acute myeloid leukaemia in first complete remission: a prospective, open-label randomised phase 3 trial. Lancet Oncol. 2012;13(10):1035-1044.

6. Monaco F, Scott BL, Chauncey TR, et al. Total body irradiation dose escalation decreases risk of progression and graft rejection after hematopoietic cell transplantation for myelodysplastic syndromes or myeloproliferative neoplasms. Haematologica. 2019;104(6):1221-1229

7. Adkins DR, DiPersio JF. Total body irradiation before an allogeneic stem cell transplantation: is there a magic dose? Curr Opin Hematol. 2008;15(6):555-560.

8. Bacigalupo A. Antilymphocyte/thymocyte globulin for graft versus host disease prophylaxis: efficacy and side effects. Bone Marrow Transplant. 2005;35(3):225-231.

9. Finke J, Bethge WA, Schmoor C, et al. Standard graft-versus-host disease prophylaxis with or without anti-T-cell globulin in haematopoietic cell transplantation from matched unrelated donors: a randomised open-label, multicentre phase 3 trial. Lancet Oncol. 2009;10(9):855864.

10. Kröger N, Solano C, Wolschke C, et al. Antilymphocyte Globulin for Prevention of chronic graft-versus-host disease. $N$ Engl J Med. 2016;374(1):43-53

11. Soiffer RJ, Kim HT, McGuirk J, et al. Prospective, randomized, doubleblind, phase III clinical trial of anti-T-lymphocyte globulin to assess impact on chronic graft-versus-host disease-free survival in patients undergoing HLA-matched unrelated myeloablative hematopoietic cell transplantation. J Clin Oncol. 2017;35(36):4003-4011.

12. Robin M, Chevret S, Koster L, et al. Antilymphocyte globulin for matched sibling donor transplantation in patients with myelofibrosis. Haematologica. 2019;104(6):1230-1236.

13. Kennedy VE, Chen H, Savani BN, et al. Optimizing antithymocyte globulin dosing for unrelated donor allogeneic hematopoietic cell transplantation based on recipient absolute lymphocyte count. Biol Blood Marrow Transplant. 2018;24(1):150-155.

14. Schmid, C, Schleuning, M, Schwerdtfeger R, et al. Long-term survival in refractory acute myeloid leukemia after sequential treatment with chemotherapy and reduced-intensity conditioning for allogeneic stem cell transplantation. Blood. 2006;108(3):1092-1099.

15. Shouval R, Bondi O, Mishan H, Shimoni A, Unger R, Nagler A. Application of machine learning algorithms for clinical predictive modeling: a data-mining approach in SCT. Bone Marrow Transplant. 2014;49(3):332-337. 